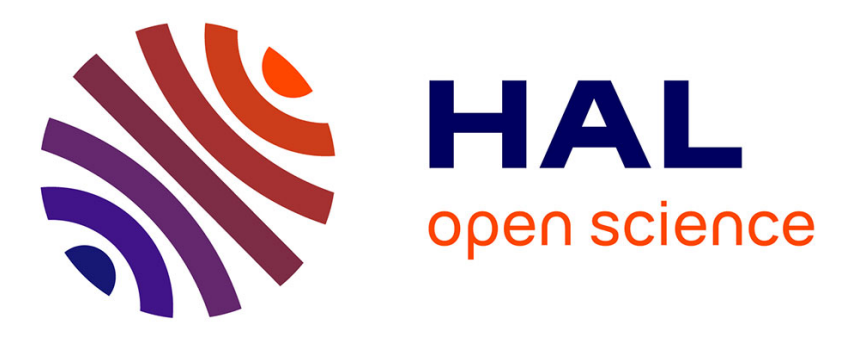

\title{
Using Deposition Rate and Substrate Temperature to Manipulate Liquid Crystal-Like Order in a Vapor-Deposited Hexagonal Columnar Glass
}

Camille Bishop, Zhenxuan Chen, Michael Toney, Harald Bock, Lian Yu, M. Ediger

\section{To cite this version:}

Camille Bishop, Zhenxuan Chen, Michael Toney, Harald Bock, Lian Yu, et al.. Using Deposition Rate and Substrate Temperature to Manipulate Liquid Crystal-Like Order in a Vapor-Deposited Hexagonal Columnar Glass. Journal of Physical Chemistry B, 2021, 125 (10), pp.2761-2770. 10.1021/acs.jpcb.0c11564 . hal-03359412

\section{HAL Id: hal-03359412 https://hal.science/hal-03359412}

Submitted on 30 Sep 2021

HAL is a multi-disciplinary open access archive for the deposit and dissemination of scientific research documents, whether they are published or not. The documents may come from teaching and research institutions in France or abroad, or from public or private research centers.
L'archive ouverte pluridisciplinaire HAL, est destinée au dépôt et à la diffusion de documents scientifiques de niveau recherche, publiés ou non, émanant des établissements d'enseignement et de recherche français ou étrangers, des laboratoires publics ou privés. 


\title{
Using Deposition Rate and Substrate Temperature to Manipulate Liquid Crystal-Like Order in a Vapor-Deposited Hexagonal Columnar Glass
}

\author{
Camille Bishop,* Zhenxuan Chen, Michael F. Toney, Harald Bock, Lian Yu, and M. D. Ediger
}

ABSTRACT: We investigate vapor-deposited glasses of a phenanthroperylene ester, known to form an equilibrium hexagonal columnar phase, and show that liquid crystal-like order can be manipulated by the choice of deposition rate and substrate temperature during deposition. We find that rate-temperature superposition (RTS) - the equivalence of lowering the deposition rate and increasing the substrate temperature-can be used to predict and control the molecular orientation in vapor-deposited glasses over a wide range of substrate temperatures $\left(0.75-1.0 T_{\mathrm{g}}\right)$. This work extends RTS to a new structural motif, hexagonal columnar liquid crystal order, which is being explored for organic electronic applications. By several metrics, including the apparent average face-to-face nearest-neighbor distance, physical vapor deposition (PVD) glasses of the phenanthroperylene ester are as ordered as the glass prepared by cooling the equilibrium liquid crystal. By other measures, the PVD glasses are less ordered than the cooled liquid crystal. We explain the difference in the maximum attainable order with the existence of a gradient in molecular mobility at the free surface of a liquid crystal and its impact upon different mechanisms of structural rearrangement. This free surface equilibration mechanism explains the success of the RTS principle and provides guidance regarding the types of order most readily enhanced by vapor deposition. This work extends the applicability of RTS to include molecular systems with a diverse range of higher-order liquid-crystalline morphologies that could be useful for new organic electronic applications.

\section{INTRODUCTION}

As the need for energy-efficient organic electronics grows, materials with new and easily tunable properties are in high demand. One class of materials that has attracted significant attention is highly anisotropic glasses. Anisotropic glasses combine the advantageous properties of crystals such as significant positional correlation $^{1,2}$ and preferential orientation $^{3}$ with properties of isotropic glasses, such as macroscopic homogeneity ${ }^{4}$ and compositional flexibility. ${ }^{5}$ This combination of properties makes anisotropic glasses uniquely suited for certain organic electronic applications such as organic lightemitting diodes (OLEDs)., ${ }^{6,7}$ Other devices, such as those based upon organic field effect transistors (OFETs), may benefit from even more highly anisotropic glasses to direct charge mobility. ${ }^{8,9}$

One way to prepare a highly anisotropic glass is by using liquid crystal mesogens. Liquid crystal (LC) glasses are highly anisotropic solids conventionally prepared from a fluid liquid crystal phase $^{10,11}$ in which the molecules form mesophases with orientational, and sometimes positional, order. Many liquid crystal morphologies exist, ranging from the simplest orientationally ordered nematic to complex mesophases with both orientational and positional order. ${ }^{12-14}$ Columnar liquid crystals are one type of highly organized morphology that has been receiving increasing attention for use in the preparation of glasses for organic electronic applications, specifically OFETs. ${ }^{8}$ In these columnar LC glasses, the disclike molecules assemble face to face into columns, and the columns further organize into mesoscale geometric motifs such as rectangular and hexagonal structures. Such glasses can be prepared by cooling from the melt, ${ }^{15}$ though it can be difficult to prepare glasses with well-aligned structures through this route. ${ }^{16}$ High levels of structural organization are important for applications in devices for directional charge mobility. ${ }^{8,17}$ Traditionally, LC systems are aligned by surface contact with an alignment layer or by application of an electric or magnetic field. ${ }^{8}$

One strategy that has been used to successfully control the order and alignment of highly organized columnar ${ }^{1,9}$ and smectic $^{19,20}$ liquid crystal glasses is physical vapor deposition (PVD). PVD can prepare LC glasses with a wide range of orientations, from molecules lying flat on a substrate to end- or edge-on arrangements. Highly organized LC and LC-like glasses with a wide range of orientations and order have been prepared by changing the substrate temperature ${ }^{1,19,20}$ and/or the rate ref2 $^{2,22}$ of deposition. More specifically, glasses with a wide range of rectangular and hexagonal columnar structures have been previously prepared by changing the substrate temperature during deposition. ${ }^{1}$ Using PVD to produce highly ordered glasses of mesogens is advantageous because the asdeposited order is independent of the underlying substrate, thereby requiring no surface modification, and PVD can be used to prepare glasses at a processing temperature below their glass transition temperature, saving energy and preserving the structure of the other layers. Additionally, vapor deposition has been shown to improve and optimize charge mobility in organic electronic columnar systems. ${ }^{9}$ The molecular alignment in PVD glasses can be explained by the surface equilibration mechanism, in which enhanced mobility at the free surface of the glass, relative to the bulk, ${ }^{23,24}$ allows newly deposited molecules to partially (or fully) equilibrate before becoming trapped in the bulk by further deposition. If the equilibrium free surface is highly anisotropic, this leads to a highly anisotropic as-deposited glass, ${ }^{25}$ as predicted by simulations ${ }^{26-28}$ and verified by experiments. ${ }^{29}$ This results in anisotropic glasses prepared from molecules with ${ }^{1,19,20}$ and without $^{3,27}$ equilibrium liquid crystal phases.

The concept of "deposition rate-substrate temperature superposition” (RTS) further extends the utility of the surface equilibration mechanism. If a structural property obeys RTS, decreasing the deposition rate and increasing the substrate temperature during deposition will alter the structure of the asdeposited glass in an equivalent manner. ${ }^{21,22}$ Increasing the substrate temperature increases the mobility of the newly deposited surface molecules, decreasing the amount of time required for them to equilibrate. Decreasing the deposition rate allows the newly deposited molecules more time at the free 
surface to equilibrate, thereby having the same effect. RTS can then be used to predict and control the glass structure over a wide range of deposition conditions. When RTS is used, it effectively reduces a two-dimensional parameter space to a single dimension and thus is a major simplification. Previous studies established the existence of RTS for both a mesogenic molecule with equilibrium liquid crystal phases ${ }^{21}$ and a nonmesogenic molecule without liquid crystal phases ${ }^{22}$ over a rather modest range of substrate temperatures - $0.94-1.00$ $T_{\mathrm{g}}$, where $T_{\mathrm{g}}$ is the glass transition temperature.

Here, we show the effect of both substrate temperature and deposition rate on the structure of a vapor-deposited phenanthroperylene ester that forms a hexagonal columnar liquid crystal glass. We show that both the temperature and the rate have a major impact on the molecular orientation, face-toface nearest-neighbor distance, and hexagonal columnar order in the vapor-deposited glass. We show that the orientational order of vapor-deposited phenanthroperylene ester obeys the rate-temperature superposition (RTS) principle over a wide range of substrate temperatures - from 0.75 to $1.0 T_{\mathrm{g}}$. Other structural parameters (apparent nearest-neighbor distance and hexagonal columnar order) are also consistent with RTS and can be precisely tuned with the proper choice of deposition conditions. Interestingly, some measures of order for the PVD glasses reach the values for the equilibrium liquid crystal, while others do not. These observations, together with the surface equilibration mechanism, indicate the presence of a gradient in mobility that controls the structural order that may be realized in vapor-deposited glasses. These new results may guide strategies to optimize the organization of columnar glasses for applications in organic electronics.

\section{METHODS}

Material Synthesis. A phenanthro[1,2,3,4,ghi]perylene$1,6,7,12,13,16$-hexacarboxylic hexaester was synthesized as described in Kelber et al. ${ }^{15}$ The melting point of its crystalline solid is $T_{\mathrm{m}}=520 \mathrm{~K}$. The supercooled liquid exhibits a hexagonal columnar phase below $492 \mathrm{~K}$ that undergoes a glass transition at $T_{\mathrm{g}}=392 \mathrm{~K} .{ }^{1}$ For the remainder of this publication, we refer to this molecule as "phenanthroperylene ester".

Vapor Deposition. Glasses of phenanthroperylene ester were prepared in a custom-built deposition chamber as outlined in a previous publication, ${ }^{1}$ using a source-to-substrate distance of $11 \mathrm{~cm}$. Samples were deposited on 1 -in. $\mathrm{Si}\langle 100\rangle$ wafers with a native oxide (Virginia Semiconductor). The Si wafers were affixed using Apiezon $\mathrm{H}$ grease to copper blocks cooled by liquid nitrogen. The temperature of the blocks was controlled using a Lakeshore 336 PID controller. The deposition rate was monitored in situ using a Quartz Crystal Microbalance (Sycon Instruments). The average sample thicknesses ranged from 35 to $120 \mathrm{~nm}$. The deposition geometry introduced a thickness gradient. The thickness at various points on the wafer was measured by ellipsometry and used to calculate the true deposition rate at each position.

Ellipsometry. An M-2000U spectroscopic ellipsometer from J.A. Woollam Co., Inc., equipped with a translational stage, was used to perform measurements on the as-deposited glasses at 21 different positions on each 1-in. wafer. For each measurement, we utilized seven angles of incidence between 45 and $75^{\circ}$. Measurements were made perpendicular to the thickness gradient across the wafer to minimize beam depolarization effects.

Ellipsometry data were collected over wavelengths from 200 to $1000 \mathrm{~nm}$. Due to a strong absorption at $\sim 350 \mathrm{~nm}$, data was modeled from 500 to $1000 \mathrm{~nm}$ using an anisotropic Cauchy model

$$
n_{z}=A_{z}+\frac{B}{\lambda^{2}}, n_{x y}=A_{x y}+\frac{B}{\lambda^{2}}
$$

in which $A_{z}, A_{x y}$ and $B$ are empirical optical constants. The difference $A_{z}-A_{x y}$ is determined in difference mode in CompleteEASE (J.A. Woollam Co.) and is equal to the birefringence $\Delta n$. Films thinner than $90 \mathrm{~nm}$ did not provide unique birefringence values, so for ellipsometry, we present data from positions on films that are thicker than $90 \mathrm{~nm}$. Ellipsometry data for a given sample is presented as a single point; the deposition rate is equal to the average of all points on samples that are thicker than $90 \mathrm{~nm}$, and the error bars for the birefringence are the standard deviations of measured values across these spots.

X-ray Scattering. Grazing-incidence wide-angle X-ray scattering (GIWAXS) was performed at Beamline 11-3 of the Stanford Synchrotron Radiation Laboratory (SSRL). The wavelength used is $0.976 \AA$, with an energy of $12.6 \mathrm{keV}$. The source-to-detector distance was $315 \mathrm{~mm}$. A fixed incidence angle of $\theta_{\text {in }}=0.12^{\circ}$ was used, above the critical angle for phenanthroperylene ester and below that of the silicon substrate. Scans were performed in a helium atmosphere for exposure times ranging from 30 to $700 \mathrm{~s}$. Structural parameters presented in this work-the orientational order parameter, nearest-neighbor distance, and hexagonal order-are quantitatively consistent for all exposure times.

Two-dimensional X-ray data were reduced using the WAXSTools plugin $^{30}$ in $\mathrm{Nika}^{31,32}$ run in the Igor Pro environment. The raw area data are processed into reciprocal space by accounting for the scattering geometry. ${ }^{33}$

The orientational order parameter $S_{\text {GIWAXs }}{ }^{34}$ was calculated to quantify the anisotropic scattering of the peak at $q \sim 1.8$ $\AA^{-1}$, which corresponds to the orientation of face-to-face nearest-neighbor interactions. All patterns were integrated from $q=1.65$ to $1.85 \AA^{-1}$ for each azimuthal angle $\chi$, with $\chi=$ $0^{\circ}$ defined along $q_{z}$. For background subtraction, similar arcs from $q=1.55$ to $1.65 \AA^{-1}$ and $q=1.85$ to $1.95 \AA^{-1}$ were integrated and averaged, and then subtracted at each value of $\chi$. The resulting $I$ vs $\chi$ curves were then fit to empirical functions and extrapolated to the regions with missing intensity, from $\chi=$ 0 to $10^{\circ}$ and $\chi=86$ to $90^{\circ}$. These procedures are shown in SI Section 1 . The resulting functions were used to calculate the sine-corrected $\left\langle\cos ^{2} \chi\right\rangle$ average of the scattering intensity

$$
\left\langle\cos ^{2} \chi\right\rangle=\frac{\int_{0}^{90} I(\chi)\left(\cos ^{2} \chi\right)(\sin \chi) \mathrm{d} \chi}{\int_{0}^{90} I(\chi)(\sin \chi) \mathrm{d} \chi}
$$

to determine $S_{\text {GIWAXS }}$

$$
S_{\text {GIWAXs }}=\frac{1}{2}\left(3\left\langle\cos ^{2} \chi\right\rangle-1\right)
$$

Values at the limit of +1 indicate that the face-to-face stacking direction is perpendicular to the substrate, and the limit of -0.5 indicates a stacking direction in the plane of the substrate.

To determine the apparent nearest-neighbor distance, we inspected the $q=1.65-1.85 \AA^{-1}$ integrations described in the previous paragraph. For each individual sample, we determined the $\chi$ range over which the intensity was at least $50 \%$ of the maximum scattering intensity; we limit our analysis to this region to report the nearest-neighbor distance for the major structural motif of the sample. We integrated the twodimensional (2D) scattering pattern over this $\chi$ range to yield $I$ vs $q$ curves. Empirical background subtraction was performed on each $I$ vs $q$ curve, and the maximum intensity determined by the first derivatives was used to determine $q_{\max }$. The apparent nearest-neighbor distance, $d_{\mathrm{nn}}$, was calculated as $d_{\mathrm{nn}}=2 \pi / q_{\max }$. More information is presented in SI Section 2 . 

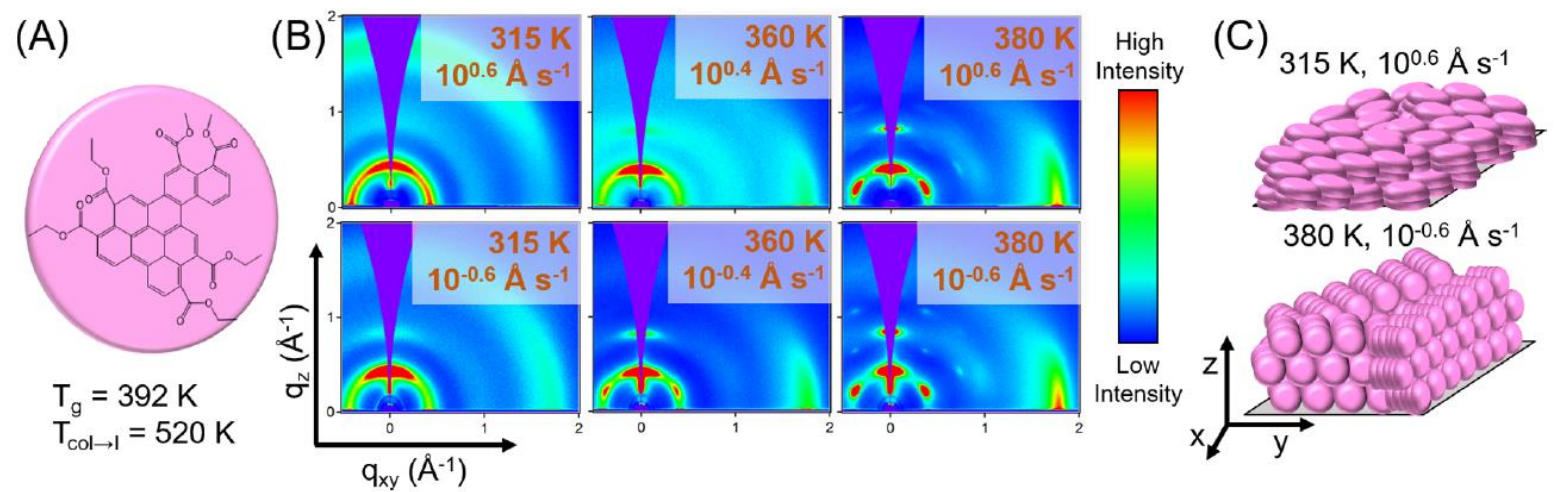

Figure 1. Two-dimensional grazing-incidence wide-angle X-ray scattering (GIWAXS) of vapor-deposited phenanthroperylene ester shows that both the substrate temperature $\left(T_{\text {sub }}\right)$ and the deposition rate change the molecular packing, including both orientational and hexagonal order. (A) Molecular structure and transition temperatures ${ }^{1}$ of phenanthroperylene ester. (B) GIWAXS patterns of phenanthroperylene ester vapor-deposited at several different substrate temperatures and rates. (C) Proposed packing motifs of the lowest $T_{\text {sub }}$ and highest deposition rate, and the highest $T_{\text {sub }}$ and lowest deposition rate sample, with real-space coordinate system is shown.

To determine the hexagonal order, the scattering intensity was integrated from $q=0.35$ to $0.55 \AA^{-1}$ at all azimuthal angles $\chi$. A constant baseline, equal to the minimum intensity at any scattering angle, was subtracted. Peaks at $\chi \sim 60^{\circ}$ were fit to Gaussians to extract the full width at half-maximum, denoted in this publication as $\Delta_{x}$

Liquid-Cooled Glass Preparation. Reference liquidcooled glasses were prepared by annealing a PVD glass in a helium atmosphere at SSRL beamline 11-3, with in situ measurements during heating and cooling. Briefly, a sample of phenanthroperylene ester vapor-deposited at $392 \mathrm{~K}\left(T_{\mathrm{g}}\right)$ and $10^{0.6} \AA / \mathrm{s}$ was heated to $457 \mathrm{~K}$ to form the equilibrium hexagonal columnar liquid crystal and then cooled to $387 \mathrm{~K}$ at approximately $2.5 \mathrm{~K} / \mathrm{min}$ (to form the liquid-cooled glass). Quantities shown for liquid-cooled glasses are measured from the glass at $387 \mathrm{~K}, 5 \mathrm{~K}$ below $T_{\mathrm{g}}$. Below $T_{\mathrm{g}}$, liquid-crystalline structural features in glasses are essentially fixed, ${ }^{11}$ meaning that the structure probed just below $T_{\mathrm{g}}$ is equivalent to that of the equilibrium liquid crystal just above $T_{\mathrm{g}}$.

\section{RESULTS}

Overview of Glassy Structures Prepared by PVD. Vapor deposition of phenanthroperylene ester at different substrate temperatures and deposition rates yields glasses with a wide range of glassy packings, as summarized qualitatively in Figure 1. The molecular structure and phase transition temperatures of phenanthroperylene ester are shown in Figure 1a. Glasses were vapor-deposited at substrate temperatures $\left(T_{\text {sub }}\right)$ from 293 to $392 \mathrm{~K}\left(0.75-1.0 T_{\mathrm{g}}\right)$ using deposition rates from $10^{-0.3}$ to $10^{0.7} \AA / \mathrm{s}$. Two-dimensional grazing-incidence wide-angle X-ray scattering (GIWAXS) patterns for a subset of these glasses are shown in Figure $1 \mathrm{~b}$, with illustrations of proposed structures for the extreme cases shown in Figure 1c. Changing $T_{\text {sub }}$ and the deposition rate during deposition produces a wide range of glassy packings, with lower deposition rates and higher substrate temperatures resulting in more ordered glasses. Given the common view that glasses are isotropic materials, we briefly explain why all of the materials in Figure $1 \mathrm{~b}$ are described here as glasses. When a liquid crystal is cooled, either a three-dimensional (3D) crystal or a glass may be formed. For both liquid crystals and isotropic liquids, glass formation occurs upon cooling when molecular motion becomes sufficiently slow (as long as crystal nucleation is avoided) and this transition is accompanied by a significant drop in the heat capacity. ${ }^{10,11}$ While an isotropic liquid yields an isotropic glass upon cooling, the glass formed from a liquid crystal inherits the anisotropic structure of the liquid-crystalline phase and different cooling rates can yield glasses with different levels of liquid-crystalline order. ${ }^{11}$ For phenanthroperylene ester, the solids shown in Figure $1 \mathrm{~b}$ form the hexagonal columnar liquid crystal when heated above $T_{\mathrm{g}}$. In addition, the most ordered glasses produced by PVD are very similar in structure to the glass produced by cooling the equilibrium liquid crystal into the glassy state (see below). Thus, the description of the materials in Figure $1 \mathrm{~b}$ as glasses is consistent with literature studies: there is no contradiction between "glass" and "liquid-crystalline order".

By changing the deposition conditions, we can prepare glasses with varying degrees of oriented hexagonal order, as seen in the X-ray scattering patterns. Hexagonal order can be observed in the GIWAXS patterns shown in Figure $1 \mathrm{~b}$ from the peak at $q \sim 0.4 \AA^{-1}(\sim 16 \AA$ in real space, the approximate diameter of the "disc" formed by one molecule). In glasses with oriented hexagonal order, local maxima of the peak occur at $\chi$ $\sim 0$ and $\pm 60^{\circ}$. Three out of the six patterns in Figure $1 \mathrm{~b}$ qualitatively show hexagonal order: those deposited at $380 \mathrm{~K}$ at both rates and the glass deposited at $360 \mathrm{~K}$ at the lower rate of $10^{-0.4} \AA / \mathrm{s}$. An illustration of the proposed oriented hexagonal order in a glass deposited at a high $T_{\text {sub }}$ and a low rate is shown in the bottom pattern of Figure 1c. Due to the grazingincidence geometry, the measurement can only distinguish hexagonal packing in-plane (perpendicular to the incident beam).

The substrate temperature and deposition rate can also be changed to prepare glasses with differing orientations of nearest-neighbor interactions. The azimuthal distribution of the broad peak at around $1.8 \AA^{-1}$ in the patterns shown in Figure $1 \mathrm{~b}$ shows the primary direction of face-to-face interactions of the disc-like molecules. This corresponds to a real-space distance of $\sim 3.5 \AA$, the interaction associated with $\pi-\pi$ molecular orbital overlap of the molecules. At a low $T_{\text {sub }}$ and a high deposition rate $\left(315 \mathrm{~K}\right.$ and $\left.10^{0.6} \AA / \mathrm{s}\right)$, the intensity is the highest nearer to $q_{z}$, suggesting that the discs primarily stack face-to-face out-of-plane, as illustrated in the top pattern of Figure 1c. As the deposition rate is lowered to $10^{-0.5} \AA / \mathrm{s}$ at the same $T_{\text {sub }}$, the intensity begins to localize along $q_{x y}$, indicating the transition to in-plane face-to-face interactions. As $T_{\text {sub }}$ is increased while the deposition rate is held nearly constant, a similar increased in-plane localization occurs, as seen going across rows in Figure 1b. Our work confirms a previous report showing that a wide range of glassy packings can be prepared from phenanthroperylene ester by changing $T_{\text {sub. }}{ }^{1}$ Here, we show, in addition, that we can use the deposition rate to prepare different glassy structures at the same $T_{\text {sub. }}$. 


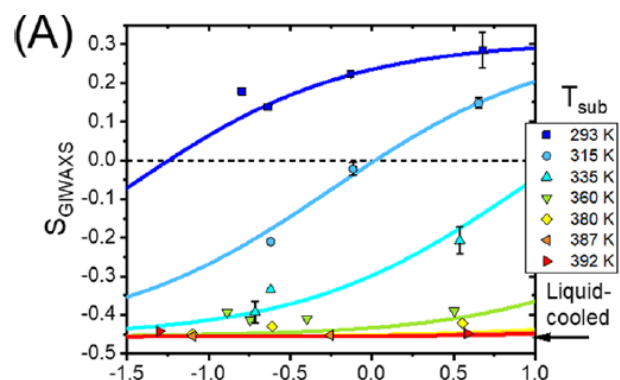

$\log \left[\right.$ deposition $\left.\operatorname{rate}\left(\AA \mathrm{s} \mathrm{s}^{-1}\right)\right]$

(B)

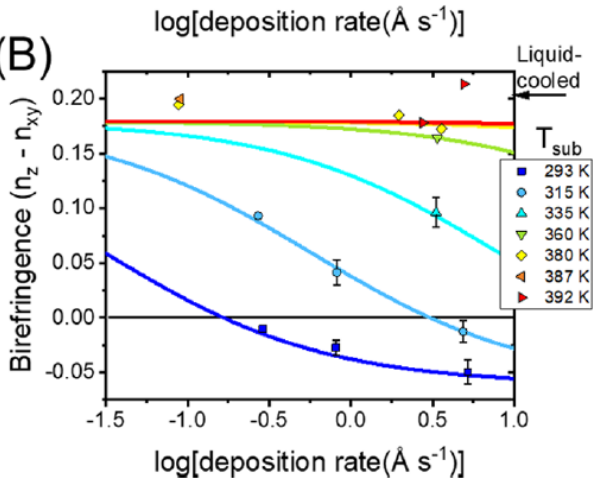

(C)

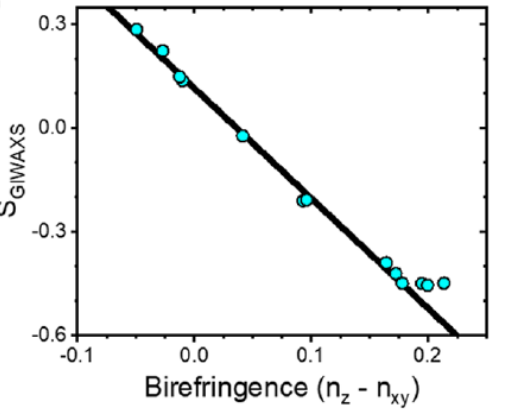

(D)

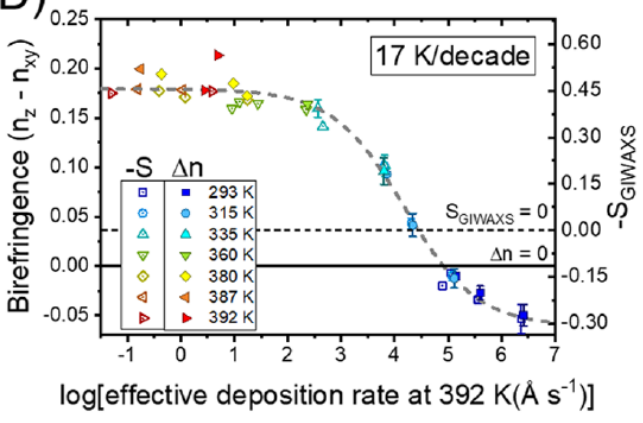

Figure 2. Measures of orientational order of vapor-deposited phenanthroperylene ester depend on both $T_{\text {sub }}$ and the deposition rate, and show deposition rate-substrate temperature superposition (RTS). (A) GIWAXS-derived orientational order parameter $S_{\text {GIwAXS }}$ for the $q \sim 1.8 \AA^{-1}$ (faceto-face nearest-neighbor interactions) peak vs the log of the deposition rate at several substrate temperatures and for the liquid-cooled glass. $S_{\text {GIWAXs }}$ ranges from 0.3 at a low $T_{\text {sub }}$ and a high rate, indicating mildly out-of-plane $\pi-\pi$ stacking, to -0.45 at a high $T_{\text {sub }}$ and a low rate, consistent with nearly perfect in-plane $\pi-\pi$ stacking (along $q_{x y}$ ). (B) Optical birefringence vs the log of the deposition rate at several substrate temperatures and for the liquid-cooled glass. A more positive birefringence, observed at a high $T_{\text {sub }}$ and a low rate, indicates a higher refractive index out-of-plane than inplane, consistent with an edge-on molecular orientation. Error bars presented are the standard deviation of the birefringence across the wafer. For points where error bars are not shown, the error bars are smaller than the size of the symbol. (C) $S_{\text {GIwAXs }}$ vs birefringence for all of the data shown in panels A and B. A linear fit is applied to all data, except for that with $S_{\text {GIWAXs }}<-0.44$, due to the uncertainty of calculating $S_{\text {GIWAXS }}$ for highly oriented samples. (D) Combined plot of $S_{\text {GIWAXs }}$ and birefringence values vs the log of the effective deposition rate at $392 \mathrm{~K}\left(T_{\mathrm{g}}\right)$. The effective deposition rate for both parameters is calculated using a shift factor of $17 \mathrm{~K}$ per decade. An empirical hyperbolic tangent fit is shown as a guide to the eye and is also shown as colored lines in panels A and B.

Orientational Order. We can quantify the orientation of nearest-neighbor interactions between adjacent phenanthroperylene ester cores using the orientational order parameter $S_{\text {GIWAXs. }} S_{\text {GIWAXS }}$ is calculated from the anisotropic scattering of the peak at $q \sim 1.8 \AA^{-1}$ as defined in the Methods section. Perfectly out-of-plane $\pi-\pi$ stacking would correspond to $S_{\text {GIWAXS }}=1$, while perfect in-plane stacking would produce $S_{\text {GIWAXs }}=-0.5$. $S_{\text {GIWAXs }}$ for phenanthroperylene ester vapordeposited at many $T_{\text {sub }}$ and deposition rates are presented in Figure 2a. As the deposition rate is lowered and $T_{\text {sub }}$ is increased, $S_{\text {GIWAXs }}$ values show that $\pi-\pi$ stacking increasingly occurs in-plane. For example, at $T_{\text {sub }}=315 \mathrm{~K}, S_{\text {GIwAXs }}$ is positive at the highest deposition rate of $10^{0.7} \AA / \mathrm{s}$, indicating out-of-plane $\pi-\pi$ stacking. As the deposition rate is lowered to $10^{-0.6} \AA / \mathrm{s}, S_{\text {GIWAXs }}$ reaches the negative value of -0.2 , with stacking that is primarily in-plane. At a constant deposition rate, glasses prepared at higher $T_{\text {sub }}$ have more negative values of $S_{\text {GIWAXS }}$, consistent with previous reports. ${ }^{1}$ At the highest $T_{\text {sub }}$ and the lowest deposition rate, $S_{\text {GIwAXs }}$ reaches -0.45 , which is very close to the limiting value of -0.5 associated with perfect in-plane $\pi-\pi$ stacking. This value of $S_{\text {GIwAXs }}$ is the same as that for the liquid-cooled glass, indicating that vapor deposition at low rates and high $T_{\text {sub }}$ results in a glass with an orientation similar to that prepared by cooling the equilibrium liquid crystal.

The optical birefringence, shown in Figure $2 b$, provides a complementary measure of orientational order that reinforces the X-ray scattering. The birefringence is sensitive to molecular orientation because the polarizability of the phenanthroperylene ester core is different in the in- and out-of-plane directions. Previous studies ${ }^{3,19}$ have shown that the birefringence can have a one-to-one correlation with the orientation of the long axis of the molecule. Similar to the data shown in
Figure $2 \mathrm{a}$, the birefringence switches sign as the deposition conditions change from a low $T_{\text {sub }}$ and a high deposition rate to a high $T_{\text {sub }}$ and a low deposition rate. At high $T_{\text {sub }} /$ low deposition rates, the birefringence is positive (the refractive index is higher out-of-plane than in-plane), indicating an edgeon molecular orientation. Since the plane of the molecule is perpendicular to the nearest-neighbor interactions, this is consistent with the negative $S_{\text {GIWAXs }}$ parameter, indicating that the interactions are primarily in-plane. We note that, in Figure $2 \mathrm{~b}$, the observed birefringence values reach the value of the liquid-cooled glass (the lines through the data show a slightly different behavior, as explained below).

The strong correlation between $S_{\text {GIwAXs }}$ and the birefringence is shown in Figure 2c. The two variables are almost perfectly anticorrelated, as expected, given that they are both sensitive to the same structural feature. We note that the lowest $S_{\text {GIWAXs }}$ points do not fit on this line. Because the molecules in these samples have stacking so strongly localized in-plane, with the scattering concentrated at $\chi>86^{\circ}$, we cannot precisely determine the value of $S_{\text {GIWAXs. }}$. We expect that the true values of the $S_{\text {GIwAXs }}$ saturate at -0.5 (the minimum possible value) at the highest $T_{\text {sub }}$ and the lowest deposition rates, even though the experimentally determined $S_{\text {GIWAXs }}$ values plateau at -0.45 .

We find that a rate-temperature superposition (RTS) successfully relates the effects of $T_{\text {sub }}$ and deposition rate on orientational order in the as-deposited phenanthroperylene ester glasses, as shown in Figure 2d. Using the anticorrelation found in Figure $2 \mathrm{c}$, we plot the $S_{\text {GIWAXs }}$ and birefringence data on linked axes. We find that the data presented in Figure 2a,b can be collapsed into a single master curve with a shift factor of $17 \mathrm{~K} /$ decade, i.e., by equating the orientational order prepared by a $17 \mathrm{~K}$ increase in $T_{\text {sub }}$ to that resulting from depositing 10 
times more slowly. This allows the calculation of an "effective deposition rate" at $392 \mathrm{~K}$, which is the deposition rate that would be required to prepare a glass with the given structure at $T_{\text {sub }}=392 \mathrm{~K}$. The temperature dependence of the relaxation time suggested by this shift factor is much weaker than that found for previous liquid-crystalline systems, consistent with a highly mobile surface, as elaborated further in the Discussion section.

One interesting feature of the data shown in Figure $2 \mathrm{~d}$ is that the zero for birefringence and $S_{\text {GIWAXs }}$ do not occur at the same effective deposition rate. Where $S_{\text {GIWAXs }}$ is equal to zero, consistent with azimuthally isotropic scattering from the $q \sim$ $1.8 \AA^{-1}$ feature, the birefringence $n_{z}-n_{x y}$ is equal to 0.04 . This may seem counterintuitive, since a truly isotropic sample would have both $S_{\text {GIWAXs }}$ and the birefringence equal to zero. The resolution to this conundrum is the observation that none of the vapor-deposited glasses are truly isotropic. For example, SI Figure S7 shows the 2D scattering pattern for the glass deposited at $315 \mathrm{~K}$ and $10^{-0.1} \AA / \mathrm{s}$. Despite the isotropic scattering of the $q \sim 1.8 \AA^{-1}$ peak for a glass that is characterized by $S_{\text {GIWAXs }}=0$, other aspects of the GIWAXS pattern are clearly anisotropic. It is possible that not all regions of the sample contribute equally to the scattering, for example, less well-ordered regions of the sample might contribute to the birefringence but not to the scattering. Other workers have reported that mildly birefringent glasses can be prepared with an isotropic molecular orientation, ${ }^{35}$ consistent with the idea that there are many dimensions to anisotropy in vapordeposited glasses.

Average Face-to-Face Nearest-Neighbor Distance. Both increasing the substrate temperature and decreasing the deposition rate decrease the face-to-face nearest-neighbor distance $d_{\mathrm{nn}}$ in vapor-deposited glasses of phenanthroperylene ester. This apparent distance is defined from the $q$-value at which the maximum scattering occurs, where $d_{\mathrm{nn}}=2 \pi / q_{\max }$. We expect that the smallest $d_{\mathrm{nn}}$ values (which occur in the most highly ordered samples) represent the $\pi$-stacking distance. In this regime, a smaller $d_{\mathrm{nn}}$ can result in an increase in the $\pi$-orbital overlap between adjacent molecules, enhancing charge mobility and performance. ${ }^{36}$ Figure $3 a$ shows a schematic illustration of $d_{\mathrm{nn}}$ in vapor-deposited phenanthroperylene ester, while $d_{\mathrm{nn}}$ is plotted against the log of the deposition rate in Figure $3 \mathrm{~b}$. At a constant deposition rate of $\sim 10^{0.5} \AA$ /s, glasses deposited at the lowest $T_{\text {sub }}$ of $293 \mathrm{~K}$ have $d_{\text {nn }}$ equal to $3.68 \AA$, while those deposited at the highest $T_{\text {sub }}$ of $392 \mathrm{~K}$ have a $d_{\mathrm{nn}}$ of $3.51 \AA-\mathrm{a} 4.5 \%$ decrease. For all $T_{\text {sub }}$ above $293 \mathrm{~K}$, a decrease in the deposition rate generally results in a decrease in $d_{\mathrm{nn}}$. The values of $d_{\mathrm{nn}}$ approach and, at the highest substrate temperature and the lowest deposition rate, are even lower than the value of the liquid-cooled glass.

Using the orientational order shift factor determined in Figure 2, we test the rate-temperature superposition (RTS) for $d_{\mathrm{nn}}$ and find that it works reasonably well, as shown in Figure 3a. The deposition rate of the glasses has been converted into an effective deposition rate at $392 \mathrm{~K}$ using a shift factor of $17 \mathrm{~K} /$ decade. Given the relatively large error bars for $d_{n n}$, we did not attempt to fit the shift factor. Thus, our analysis indicates that the $d_{\mathrm{nn}}$ values are consistent with the shift factor found for molecular orientation.

The correlation length associated with the nearest-neighbor peak depends upon deposition conditions in a manner that is qualitatively consistent with data in Figures 2 and 3. We find that the correlation length ranges from about $1.5 \mathrm{~nm}(\sim 4$ molecules) for the sample deposited at the lowest $T_{\text {sub }}$ and the highest rate up to about $3.3 \mathrm{~nm}$ ( $\sim 9$ molecules) for the sample deposited at the highest $T_{\text {sub }}$ and the lowest rate.
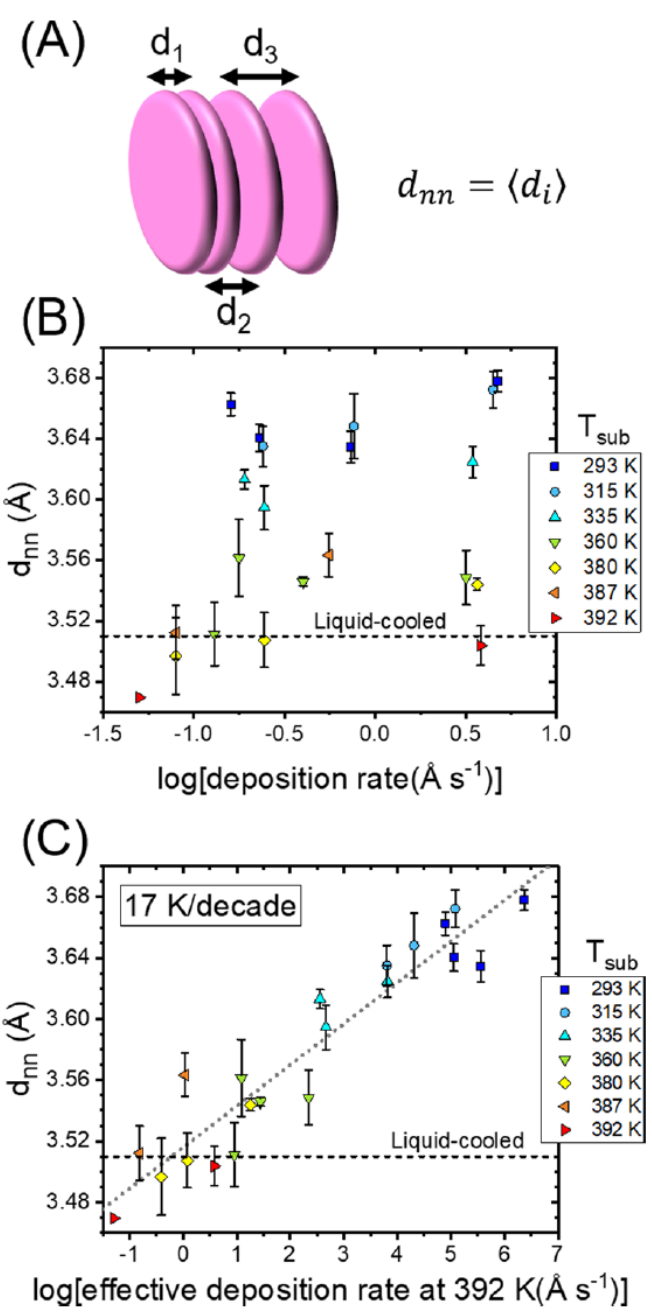

Figure 3. The apparent average nearest-neighbor distance $d_{\mathrm{nn}}$ is significantly modified by the deposition rate and the substrate temperature and can be described using deposition rate-substrate temperature superposition (RTS). (A) Illustration quantifying $d_{\mathrm{nn}}$ the average distance between two adjacent disc-like molecules in the vapor-deposited glass. (B) $d_{\mathrm{nn}}$ of vapor-deposited phenanthroperylene ester as a function of the deposition rate at several $T_{\text {sub }}$. Generally, $d_{\mathrm{nn}}$ decreases at a higher $T_{\text {sub }}$ and lower deposition rates. The value of $d_{\mathrm{nn}}$ for the liquid-cooled glass is also indicated. Error bars show the standard deviation of the peak position found from the measurements at distinct spots on the sample. (C) RTS successfully describes $d_{\mathrm{nn}}$ using a shift factor of $17 \mathrm{~K} /$ decade, the same shift factor used for molecular orientation in Figure 2. A linear fit to all data points is shown as a guide to the eye.

Hexagonal Order. Similar to the molecular orientation and the apparent nearest-neighbor distance, we find that glasses with a higher degree of in-plane hexagonal order are obtained by decreasing the deposition rate and increasing $T_{\text {sub }}$. To quantify the hexagonal order, we integrate the peak at $q \sim$ $0.4 \AA^{-1}$ (from $q=0.35$ to $0.55 \AA^{-1}$ ) for every azimuthal angle $\chi$, as illustrated schematically in Figure 4a. Example integrations are shown in Figure $4 \mathrm{~b}$. There is no intensity data in the range $\chi= \pm 8^{\circ}$ due to the scattering geometry, ${ }^{33}$ though all samples show a peak near this area regardless of the extent of hexagonal order. As $T_{\text {sub }}$ is increased and the deposition rate is decreased, peaks at $\chi \sim 60^{\circ}$ grow in size and narrow in width. We subtract a linear background and fit these peaks to a Gaussian function, using the full width at halfmaximum, denoted $\Delta_{\chi}$, as a measure of hexagonal order. Figure $4 \mathrm{c}$ shows $\Delta_{\chi}$ values as a function of the log of the deposition rate at several $T_{\text {sub }}$. Glasses deposited below $360 \mathrm{~K}$ do not show any in-plane hexagonal order and are not included in Figure $4 \mathrm{c}$. At all $T_{\text {sub }}$ studied, depositing more slowly prepares 


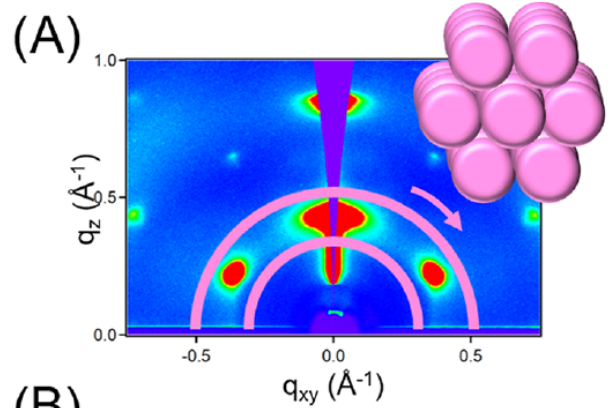

(B)

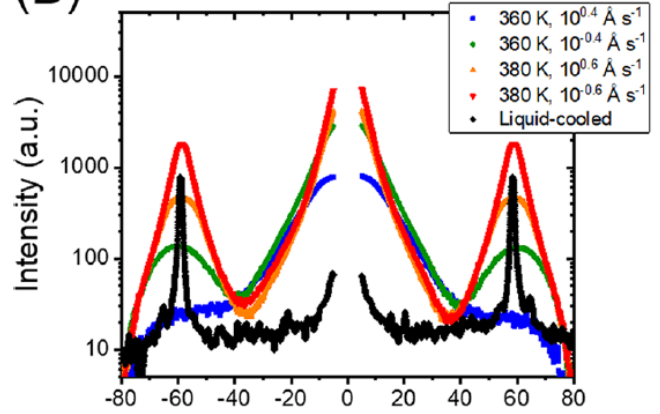

(C)
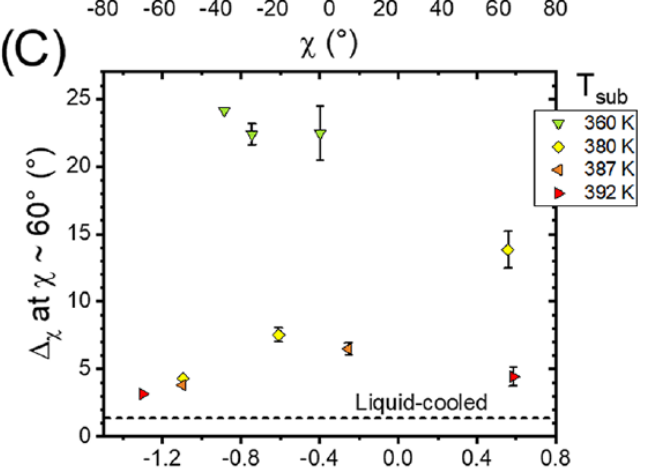

(D)

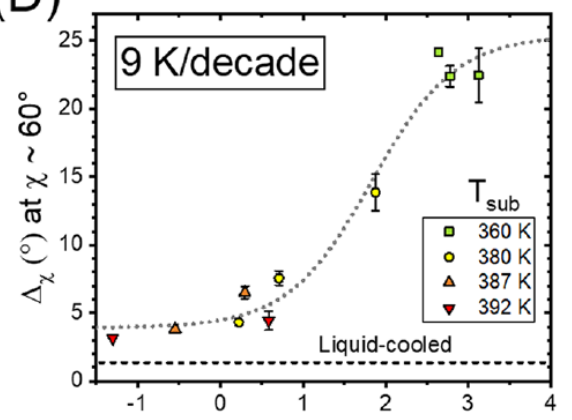

$\log \left[\right.$ effective deposition rate at $\left.392 \mathrm{~K}\left(\AA^{-1} \mathrm{~s}^{-1}\right)\right]$

Figure 4. Azimuthal integrations of the $q \sim 0.4 \AA^{-1}$ peak reveal that the in-plane hexagonal order depends upon $T_{\text {sub }}$ and the deposition rate, and can be described by RTS. (A) Illustration of the area in the 2D GIWAXS pattern used to determine hexagonal order, and a schematic of a highly ordered system. Intensity is integrated from $q=$ 0.35 to $0.55 \AA^{-1}$ at every value of the azimuthal angle $\chi$. (B) Scattering intensity for selected samples as a function of $\chi$ for vapor-deposited and liquid-cooled glasses. (C) FWHM $\left(\Delta_{\chi}\right)$ of the peak at $\chi \sim 60^{\circ}$ as a function of the deposition rate for all samples showing hexagonal order. Generally, increasing $T_{\text {sub }}$ and lowering the deposition rate result in a smaller $\Delta_{\chi}$, consistent with increasingly perfected in-plane hexagonal order. (D) Hexagonal order can be described using RTS. $\Delta_{\chi}$, the full width at half-maximum (FWHM) of the hexagonal order peak at $\chi \sim 60^{\circ}$, is plotted against the log of the effective deposition rate at $392 \mathrm{~K}$ calculated using a shift factor of $9 \mathrm{~K}$ per decade in rate. An empirical hyperbolic tangent fit is shown as a guide to the eye.

glasses with greater in-plane hexagonal order $\left(\right.$ smaller $\left.\Delta_{\chi}\right)$. Generally, increasing $T_{\text {sub }}$ also increases the hexagonal order. All glasses prepared by vapor deposition have less in-plane hexagonal order than the glass prepared by liquid cooling, with the smallest value of $\Delta_{\chi}$ being $3^{\circ}$ as compared to the liquidcooled value of $1^{\circ}$. Due to the grazing-incidence geometry, the intensity of the $\chi \sim 60^{\circ}$ peaks is sensitive to both the degree of hexagonal order within a domain and the degree to which the hexagonal columns lie in the substrate plane. Additionally, we find that the sample deposited at the highest effective deposition rate has a correlation length of $5.1 \mathrm{~nm}$ or 3-4 molecular diameters. The sample deposited at the lowest effective rate has a correlation length of $33 \mathrm{~nm}$, or $\sim 20$ molecular diameters, suggesting a highly organized sample. We also calculate the edge-to-edge disc spacing of this hexagonal peak at around $q \sim 0.4 \AA^{-1}$, shown in SI Section 5 .

We test the RTS principle for hexagonal order and find that $\Delta_{\chi}$ superposes successfully, but with a different shift factor than that found for the orientational order and nearest-neighbor distance. In Figure $4 \mathrm{~d}$, we show $\Delta_{\chi}$ as a function of the effective deposition rate calculated from a superposition factor of $9 \mathrm{~K}$ per decade. While the existence of multiple shift factors may seem surprising, this was previously reported for orientational and positional order in a vapor-deposited smectic liquid crystal, ${ }^{21}$ and attributed to the existence of multiple relaxation processes at the free surface. Below, we show that these distinct shift factors provide insight into the surface equilibration process during PVD.

\section{DISCUSSION}

Deposition Rate-Substrate Temperature Superposition of Orientational Order. This work expands the range of systems for which RTS has been shown to describe orientational order in vapor-deposited glasses. The effects of the deposition rate and $T_{\text {sub }}$ on the structure of three vapordeposited systems have now been systematically studied: the rod-like molecules itraconazole (a smectic liquid crystal) and posaconazole (with no liquid crystal phases), and now the disclike phenanthroperylene ester, a hexagonal columnar liquid crystal. In contrast to previous work, phenanthroperylene ester is a potential organic photovoltaic material with favorable electronic properties. ${ }^{15}$ For all three of these systems, the molecules in the vapor-deposited glass orient edge-on with respect to the substrate when prepared at a higher $T_{\text {sub }}$ and/or a lower deposition rate. As these conditions maximize equilibration at the free surface during deposition, this result is consistent with the surface equilibration mechanism for PVD glasses (since an edge-on orientation is the typical equilibrium liquid crystal orientation at the free surface). ${ }^{18}$

This work also expands the range of substrate temperature over which RTS for orientational order can be expected to work $\left(0.75-1.00 T_{\mathrm{g}}\right)$. Previous work by Bishop et al. covered a more modest range of substrate temperatures from 0.94 to 1.00 $T_{\mathrm{g}}{ }^{21,22}$ Previous work by Yokoyama and co-workers, while not explicitly investigating RTS, found different birefringence values for OLED materials TPT1, 2-TNATA, and TPD deposited at different rates at a single substrate temperature (0.70, 0.77, and $0.88 T_{\mathrm{g}}$, respectively). ${ }^{6,37}$ Additionally, Bagchi et al. found differing translational order at two deposition rates at several substrate temperatures for $\mathrm{Alq}_{3}$, a spheroidal OLED molecule. ${ }^{27}$ The results from these studies on OLED molecules together with the present study suggest that RTS may be applied systematically to technologically relevant materials over a wide range of deposition conditions.

Comparison of PVD Glasses with Glasses Prepared by Cooling the Equilibrium Liquid Crystal. To evaluate the structures of PVD glasses of phenanthroperylene ester, it is convenient to use the equilibrium liquid crystal as a reference state representing perfect liquid crystal order. For two of the types of structural order characterized in this work, PVD glasses can achieve the same level of structural perfection as glasses prepared by cooling the equilibrium liquid crystal. For 
example, glasses deposited at sufficiently high $T_{\text {sub }}$ and a sufficiently low rate reach the values for the apparent nearestneighbor distance, $d_{\mathrm{nn}}$, and the orientational order that result from cooling the equilibrium liquid crystal, as measured by $S_{\text {GIWAXs }}$ and birefringence. The PVD glass deposited at the lowest rate at $392 \mathrm{~K}$ exhibited values of $d_{\mathrm{nn}}$ that are even smaller than that found for the equilibrium liquid crystal; this glass presumably is more equilibrated than our reference sample, which has a structure that resembles the liquid crystal at a temperature of around $392 \mathrm{~K}$, where molecular motions arrest upon cooling. However, even at the lowest effective deposition rate studied, the hexagonal order as measured by $\Delta_{\chi}$ never reaches the equilibrium value of $1.5^{\circ}$, instead reaching a minimum value of $3^{\circ}$ by vapor deposition. The behavior of the different structural parameters can be understood using the surface equilibration mechanism, as we discuss below.

The Surface Equilibration Mechanism and a Gradient in Mobility. Organic glasses can have highly mobile surfaces that allow partial or full equilibration during PVD even when the substrate temperature is below $T_{\mathrm{g} \cdot}{ }^{38-40}$ By this means, glasses with greater density and much higher kinetic stability can be prepared. ${ }^{23}$ Since equilibration during PVD occurs near an interface, anisotropic glasses are often obtained. For glasses of phenanthroperylene ester prepared at a high $T_{\text {sub }}$ and a low deposition rate, conditions that favor equilibration, strongly edge-on packing relative to the free surface is observed. We interpret this to mean that edge-on packing is preferred at the free surface of the equilibrium liquid crystal, and this is consistent with experimental results for other disc-like liquid crystals. $^{41,42}$

The surface equilibration mechanism can be used to rationalize why PVD can more readily perfect certain types of liquid-crystalline order than others. Computer simulations and experiments indicate that there is a strong gradient in mobility near the free surface of an organic glass. Dynamics are most accelerated at the free surface and somewhat slower for a few molecular diameters below the interface, with bulk-like dynamics ${ }^{43-45}$ being achieved at a greater distance (often thought to be $\sim 5 \mathrm{~nm}) .{ }^{24}$ We speculate that the measures of order that fully attain equilibrium values during vapor deposition of phenanthroperylene ester, orientation and nearest-neighbor distance, require rearrangement only in the top monolayer $(1.5 \mathrm{~nm})$ where mobility is greatest. We imagine that hexagonal order, which requires registry across at least two molecular layers $(3 \mathrm{~nm})$, does not reach equilibrium for the same deposition conditions because rearrangement would be required deeper into the film where the dynamics are slower. We note that the region of high mobility $(\sim 5 \mathrm{~nm})$ near the free surface is much smaller than the coherence length for hexagonal packing (up to $30 \mathrm{~nm}$ ), and so we infer that it is possible to prepare a thick region of an ordered hexagonal structure by equilibrating only a few layers at a time during deposition.

The shift factor for hexagonal order is smaller than that observed for the nearest-neighbor distance and the orientational order, and this supports the idea that hexagonal order requires equilibration to a greater depth during deposition. The gradient in mobility near the surface of an organic glass is associated with a gradient in activation energies. Depending upon the system and the temperature, activation barriers can be 2 to 5 times smaller at the surface than in the bulk. ${ }^{38,45} \mathrm{We}$ can relate the temperature dependence of the shift factors to this gradient in activation energies. For the orientational ordering of phenanthroperylene ester, a shift factor of $17 \mathrm{~K} /$ decade indicates an $E_{\mathrm{a}}$ of $110 \mathrm{~kJ} / \mathrm{mol}$, while the $9 \mathrm{~K} /$ decade shift factor for the hexagonal ordering indicates an $E_{\mathrm{a}}$ of 210 $\mathrm{kJ} / \mathrm{mol}$. This greater activation energy for the hexagonal ordering suggests that the rearrangement occurs further into the bulk of the film, where barriers to rearrangement are higher. $^{44}$

The gradient in activation energies near the free surface also allows us to understand results in the literature for PVD glasses of itraconazole, a smectic liquid crystal. Bishop et al. found different shift factors for the orientational and translational order in itraconazole. ${ }^{21}$ The shift factor for the orientational order yields an activation energy of $390 \mathrm{~kJ} / \mathrm{mol}$, while that for the presumably more long-range process of smectic layering is $920 \mathrm{~kJ} / \mathrm{mol}$. In comparison with itraconazole, the substantially lower activation energies reported here for phenanthroperylene ester are consistent with a highly mobile free surface and indicate that the deposition rate is a much more potent factor for controlling structural order.

Versatility of PVD for Preparing Highly Anisotropic Glasses. PVD of phenanthroperylene ester provides an efficient route to highly anisotropic glasses with desirable properties. In comparison to slow cooling from the equilibrium liquid crystal, PVD offers advantages of low-temperature processing and uniform orientation. For example, a glass can be prepared with the equilibrium value for $d_{\mathrm{nn}}$ at $T_{\text {sub }}=360 \mathrm{~K}$ by depositing sufficiently slowly. As equilibrium processing requires transformation in the melt (well above $392 \mathrm{~K}$ ), PVD enables a much lower processing temperature to be used. This is very advantageous in a multilayer manufacturing process in which high temperatures may be deleterious to the other components in a device. In addition, PVD can prepare films with uniform orientation, even for films that are too thick to be aligned using alignment layers in confinement. ${ }^{18}$ Even if PVD at a given $T_{\text {sub }}$ /deposition rate condition does not lead to the desired equilibrium property, the as-deposited glass may have sufficient alignment of the molecules to achieve the desired orientation with limited subsequent annealing near $T_{g}$; if the same glass were prepared by spin-coating or heating a powder, followed by cooling from the melt, it would likely take much longer to reach the desired orientation, if it can be achieved at all.

An additional advantage of preparing glasses by PVD is access to a large range of structures that are likely not accessible by any other practical route. For phenanthroperylene ester, a wide range of glassy structures are possible, based upon the four structural metrics presented here. We find a wide range of molecular orientations, with nearest-neighbor stacking varying from mildly out-of-plane to strongly in-plane. The nearest-neighbor distance varies from 3.47 to $3.70 \AA$ and likely provides an opportunity to tune charge mobility. ${ }^{36} \Delta_{\gamma}$, the measure of hexagonal order in this work, also varies widely from 3 to $24^{\circ}$. It is possible that glasses with some of these outof-equilibrium properties could also be prepared by fast quenching from the melt, as has been performed for smectic liquid crystals. ${ }^{11}$ However, the required cooling rates are likely incompatible with other requirements in the context of device manufacture.

Additional experimental techniques will be needed to characterize the domain structure of the columns of phenanthroperylene ester in PVD glasses, including their length. Due to the deposition geometry, columns propagate in all in-plane directions. ${ }^{1}$ Atomic force microscopy (AFM) could be used in conjunction with high-resolution electron microscopy ${ }^{46}$ to map the domain morphology of samples. One might expect that domains would be longer in samples that are deposited more slowly and at higher substrate temperatures; these longer domains would be expected to transport charge more effectively. 


\section{CONCLUSIONS}

Over a wide range of deposition conditions, the glassy packing of vapor-deposited phenanthroperylene ester can be controlled by changing the deposition rate and the substrate temperature. Deposition at higher substrate temperatures and lower rates results in glasses with more edge-on packing, a smaller face-toface nearest-neighbor distance (likely resulting in greater $\pi-\pi$ overlap), and more hexagonal columnar order. These types of order can be understood and controlled using the concept of "rate-temperature superposition", or RTS. The success of RTS can be explained by the surface equilibration mechanism, in which structure in the vapor-deposited glass is a consequence of partial equilibration at the free surface during deposition; the same equilibration can occur either as a result of increasing the substrate temperature or decreasing the deposition rate. A gradient in mobility from the free surface explains why the different types of order investigated in this work can be perfected to various degrees by vapor deposition. This is the first time the concept of RTS, found initially in model smectic systems, has been extended to a columnar liquid crystal morphology that shows promising electrical properties. ${ }^{15}$ For the phenanthroperylene ester investigated here, RTS works over a wide range of substrate temperatures (from $T_{\mathrm{g}}$ down to $0.75 T_{\mathrm{g}}$ ). We therefore show how substrate temperature and deposition rate can be selected to optimize materials for new organic electronic applications.

\section{AUTHOR INFORMATION}

\section{Corresponding Author}

Camille Bishop - Department of Chemistry, University of Wisconsin-Madison, Madison, Wisconsin 53706, United States; ๑orcid.org/0000-0002-2889-1752;

Email: camille.bishop@nist.gov

\section{Authors}

Zhenxuan Chen - School of Pharmacy, University of Wisconsin-Madison, Madison, Wisconsin 53705, United States

Michael F. Toney - Department of Chemical and Biological Engineering, University of Colorado Boulder, Boulder,

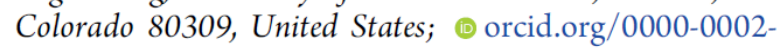
7513-1166

Harald Bock - Centre de Recherche Paul Pascal, CNRS \& Université de Bordeaux, 33600 Pessac, France

Lian Yu - Department of Chemistry, University of Wisconsin-Madison, Madison, Wisconsin 53706, United States; School of Pharmacy, University of WisconsinMadison, Madison, Wisconsin 53705, United States; (1) orcid.org/0000-0002-4253-5658

M. D. Ediger - Department of Chemistry, University of Wisconsin-Madison, Madison, Wisconsin 53706, United States; ○ orcid.org/0000-0003-4715-8473

\section{ACKNOWLEDGMENTS}

We gratefully acknowledge LG Chem for support of this research. Additional support was received from NSF through the University of Wisconsin Materials Research Science and Engineering Center (Grant DMR-1720415), and through Grant DMR-1904601. Use of the Stanford Synchrotron Radiation Lightsource, SLAC National Accelerator Laboratory, is supported by the US Department of Energy, Office of Science, Office of Basic Energy Sciences under Contract DEAC02-76SF00515.

\section{REFERENCES}

(1) Gujral, A.; Gómez, J.; Ruan, S.; Toney, M. F.; Bock, H.; Yu, L.; Ediger, M. D. Vapor-Deposited Glasses with Long-Range Columnar Liquid Crystalline Order. Chem. Mater. 2017, 29, 9110-9119.

(2) Eccher, J.; Faria, G. C.; Bock, H.; Von Seggern, H.; Bechtold, I. $\mathrm{H}$. Order Induced Charge Carrier Mobility Enhancement in Columnar Liquid Crystal Diodes. ACS Appl. Mater. Interfaces 2013, 5, 11935-11943.

(3) Dalal, S. S.; Walters, D. M.; Lyubimov, I.; de Pablo, J. J.; Ediger, M. D. Tunable molecular orientation and elevated thermal stability of vapor-deposited organic semiconductors. Proc. Natl. Acad. Sci. U.S.A. 2015, 112, 4227-4232.

(4) Bolognesi, A.; Berliocchi, M.; Manenti, M.; Carlo, A. D.; Lugli, P.; Lmimouni, K.; Dufour, C. Effects of grain boundaries, fielddependent mobility, and interface trap states on the electrical characteristics of pentacene TFT. IEEE Trans. Electron Devices 2004, 51, 1997-2003.

(5) Schmidt, T. D.; Lampe, T.; Sylvinson, M. R. D.; Djurovich, P. I.; Thompson, M. E.; Brütting, W. Emitter Orientation as a Key Parameter in Organic Light-Emitting Diodes. Phys. Rev. Appl. 2017, 8, No. 037001.

(6) Yokoyama, D. Molecular orientation in small-molecule organic light-emitting diodes. J. Mater. Chem. 2011, 21, 19187-19202.

(7) Ràfols-Ribé, J.; Will, P.-A.; Hänisch, C.; Gonzalez-Silveira, M.; Lenk, S.; Rodríguez-Viejo, J.; Reineke, S. High-performance organic light-emitting diodes comprising ultrastable glass layers. Sci. Adv. 2018, 4, No. eaar8332.

(8) O’Neill, M.; Kelly, S. M. Ordered materials for organic electronics and photonics. Adv. Mater. 2011, 23, 566-584.

(9) Eccher, J.; Zajaczkowski, W.; Faria, G. C.; Bock, H.; von Seggern, H.; Pisula, W.; Bechtold, I. H. Thermal Evaporation versus SpinCoating: Electrical Performance in Columnar Liquid Crystal OLEDs. ACS Appl. Mater. Interfaces 2015, 7, 16374-16381.

(10) Suga, H.; Seki, S. Thermodynamic investigation on glassy states of pure simple compounds. J. Non-Cryst. Solids 1974, 16, 171-194.

(11) Teerakapibal, R.; Huang, C.; Gujral, A.; Ediger, M. D.; Yu, L. Organic Glasses with Tunable Liquid-Crystalline Order. Phys. Rev. Lett. 2018, 120, No. 055502.

(12) Sorai, M.; Seki, S. Glassy Liquid Crystal of the Nematic Phase of N-(o-Hydroxy-p-methoxybenzylidene)-p-butylaniline. Bull. Chem. Soc. Jpn. 1971, 44, 2887.

(13) Tsuji, K.; Sorai, M.; Seki, S. New Finding of Glassy Liquid Crystal - a Non-equilibrium State of Cholesteryl Hydrogen Phthalate. Bull. Chem. Soc. Jpn. 1971, 44, 1452.

(14) Farias, G.; Simeão, D. S.; Moreira, T. S.; dos Santos, P. L.; Bentaleb, A.; Girotto, E.; Monkman, A. P.; Eccher, J.; Durola, F.; Bock, H.; et al. An unusual plank-shaped nematogen with a graphene nanoribbon core. J. Mater. Chem. C 2019, 7, 12080-12085.

(15) Kelber, J.; Achard, M.-F.; Durola, F.; Bock, H. Distorted Arene Core Allows Room-Temperature Columnar Liquid-Crystal Glass with Minimal Side Chains. Angew. Chem., Int. Ed. 2012, 51, 5200-5203.

(16) Al-Lawati, Z. H.; Bushby, R. J.; Evans, S. D. Alignment of a Columnar Hexagonal Discotic Liquid Crystal on Self-Assembled Monolayers. J. Phys. Chem. C 2013, 117, 7533-7539.

(17) Bushby, R. J.; Lozman, O. R. Discotic liquid crystals 25 years on. Curr. Opin. Colloid Interface Sci. 2002, 7, 343-354.

(18) Jerome, B. Surface effects and anchoring in liquid crystals. Rep. Prog. Phys. 1991, 54, 391-451.

(19) Gómez, J.; Jiang, J.; Gujral, A.; Huang, C.; Yu, L.; Ediger, M. D. Vapor deposition of a smectic liquid crystal: highly anisotropic, homogeneous glasses with tunable molecular orientation. Soft Matter 2016, 12, 2942-2947.

(20) Gujral, A.; Gómez, J.; Jiang, J.; Huang, C.; O’Hara, K. A.; Toney, M. F.; Chabinyc, M. L.; Yu, L.; Ediger, M. D. Highly Organized Smectic-like Packing in Vapor-Deposited Glasses of a Liquid Crystal. Chem. Mater. 2017, 29, 849-858.

(21) Bishop, C.; Gujral, A.; Toney, M. F.; Yu, L.; Ediger, M. D. Vapor-Deposited Glass Structure Determined by Deposition RateSubstrate Temperature Superposition Principle. J. Phys. Chem. Lett. 2019, 10, 3536-3542.

(22) Bishop, C.; Li, Y.; Toney, M. F.; Yu, L.; Ediger, M. D. Molecular Orientation for Vapor-Deposited Organic Glasses Follows Rate-Temperature Superposition: The Case of Posaconazole. J. Phys. Chem. B 2020, 124, 2505-2513. 
(23) Swallen, S. F.; Kearns, K. L.; Mapes, M. K.; Kim, Y. S.; McMahon, R. J.; Ediger, M. D.; Wu, T.; Yu, L.; Satija, S. Organic Glasses with Exceptional Thermodynamic and Kinetic Stability. Science 2007, 315, 353-356.

(24) Chen, Y.; Chen, Z.; Tylinski, M.; Ediger, M. D.; Yu, L. Effect of molecular size and hydrogen bonding on three surface-facilitated processes in molecular glasses: Surface diffusion, surface crystal growth, and formation of stable glasses by vapor deposition. J. Chem. Phys. 2019, 150, No. 024502.

(25) Harrowell, P. Orientationally ordered glasses via controlled deposition. Proc. Natl. Acad. Sci. U.S.A. 2019, 116, 21341-21342.

(26) Lyubimov, I.; Antony, L.; Walters, D. M.; Rodney, D.; Ediger, M. D.; de Pablo, J. J. Orientational anisotropy in simulated vapordeposited molecular glasses. J. Chem. Phys. 2015, 143, No. 094502.

(27) Bagchi, K.; Jackson, N. E.; Gujral, A.; Huang, C.; Toney, M. F.; Yu, L.; de Pablo, J. J.; Ediger, M. D. Origin of Anisotropic Molecular Packing in Vapor-Deposited Alq3 Glasses. J. Phys. Chem. Lett. 2019, 10, 164-170.

(28) Moore, A. R; Huang, G.; Wolf, S.; Walsh, P. J.; Fakhraai, Z.; Riggleman, R. A. Effects of microstructure formation on the stability of vapor-deposited glasses. Proc. Natl. Acad. Sci. U.S.A. 2019, 116, $5937-5942$.

(29) Bishop, C.; Thelen, J. L.; Gann, E.; Toney, M. F.; Yu, L.; DeLongchamp, D. M.; Ediger, M. D. Vapor deposition of a nonmesogen prepares highly structured organic glasses. Proc. Natl. Acad. Sci. U.S.A. 2019, 116, 21421-21426.

(30) Oosterhout, S. D.; Savikhin, V.; Zhang, J.; Zhang, Y.; Burgers, M. A.; Marder, S. R.; Bazan, G. C.; Toney, M. F. Mixing Behavior in Small Molecule:Fullerene Organic Photovoltaics. Chem. Mater. 2017, 29, 3062-3069.

(31) Ilavsky, J. Nika: software for two-dimensional data reduction. J. Appl. Crystallogr. 2012, 45, 324-328.

(32) Zhang, F.; Ilavsky, J.; Long, G. G.; Quintana, J. P. G.; Allen, A. J.; Jemian, P. R. Glassy Carbon as an Absolute Intensity Calibration Standard for Small-Angle Scattering. Metall. Mater. Trans. A 2010, 41, $1151-1158$.

(33) Baker, J. L.; Jimison, L. H.; Mannsfeld, S.; Volkman, S.; Yin, S.; Subramanian, V.; Salleo, A.; Alivisatos, A. P.; Toney, M. F. Quantification of Thin Film Crystallographic Orientation Using Xray Diffraction with an Area Detector. Langmuir 2010, 26, 91469151.

(34) Gujral, A.; O’Hara, K. A.; Toney, M. F.; Chabinyc, M. L.; Ediger, M. D. Structural characterization of vapor-deposited glasses of an organic hole transport material with X-ray scattering. Chem. Mater. 2015, 27, 3341-3348.

(35) Liu, T.; Exarhos, A. L.; Alguire, E. C.; Gao, F.; SalamiRanjbaran, E.; Cheng, K.; Jia, T.; Subotnik, J. E.; Walsh, P. J.; Kikkawa, J. M.; et al. Birefringent Stable Glass with Predominantly Isotropic Molecular Orientation. Phys. Rev. Lett. 2017, 119, No. 095502.

(36) Coropceanu, V.; Cornil, J.; da Silva Filho, D. A.; Olivier, Y.; Silbey, R.; Brédas, J.-L. Charge Transport in Organic Semiconductors. Chem. Rev. 2007, 107, 926-952.

(37) Shibata, M.; Sakai, Y.; Yokoyama, D. Advantages and disadvantages of vacuum-deposited and spin-coated amorphous organic semiconductor films for organic light-emitting diodes. J. Mater. Chem. C 2015, 3, 11178-11191.

(38) Yu, L. Surface mobility of molecular glasses and its importance in physical stability. Adv. Drug Delivery Rev. 2016, 100, 3-9.

(39) Samanta, S.; Huang, G.; Gao, G.; Zhang, Y.; Zhang, A.; Wolf, S.; Woods, C. N.; Jin, Y.; Walsh, P. J.; Fakhraai, Z. Exploring the Importance of Surface Diffusion in Stability of Vapor-Deposited Organic Glasses. J. Phys. Chem. B 2019, 123, 4108-4117.
(40) Berthier, L.; Charbonneau, P.; Flenner, E.; Zamponi, F. Origin of Ultrastability in Vapor-Deposited Glasses. Phys. Rev. Lett. 2017, 119, No. 188002.

(41) De Cupere, V.; Tant, J.; Viville, P.; Lazzaroni, R.; Osikowicz, W.; Salaneck, W. R.; Geerts, Y. H. Effect of Interfaces on the Alignment of a Discotic Liquid-Crystalline Phthalocyanine. Langmuir 2006, 22, 7798-7806.

(42) Grelet, E.; Bock, H. Control of the orientation of thin open supported columnar liquid crystal films by the kinetics of growth. Europhys. Lett. 2006, 73, 712-718.

(43) Ruan, S.; Zhang, W.; Sun, Y.; Ediger, M. D.; Yu, L. Surface diffusion and surface crystal growth of tris-naphthyl benzene glasses. J. Chem. Phys. 2016, 145, No. 064503.

(44) Mirigian, S.; Schweizer, K. S. Theory of activated glassy relaxation, mobility gradients, surface diffusion, and vitrification in free standing thin films. J. Chem. Phys. 2015, 143, No. 244705.

(45) Zhang, Y.; Fakhraai, Z. Decoupling of surface diffusion and relaxation dynamics of molecular glasses. Proc. Natl. Acad. Sci. U.S.A. 2017, 114, 4915-4919.

(46) Panova, O.; Ophus, C.; Takacs, C. J.; Bustillo, K. C.; Balhorn, L.; Salleo, A.; Balsara, N.; Minor, A. M. Diffraction imaging of nanocrystalline structures in organic semiconductor molecular thin films. Nat. Mater. 2019, 18, 860-865. 